\title{
IV. AUTHORS OF PAPERS READ BEFORE THE AMERICAN MATHEMATICAL SOCIETY, 1914-1924
}

Adams, C. R., XXX, 211.

Alexander, J. W., XXI, 272, 484; XXII, 266, 483; XXVI, 52, 338, 338; XXVIII, 10,$10 ;$ XXIX, 116, 148; XXX, 10, 10, 299.

Alger, P. L., XXV, 242; XXVI, 244.

Allardice, R. E., XXV, 393.

Allen, E. F., XXX, 99.

Allen, E. S., XXVI, 52; XXIX, 106.

Allen, F. E., XXV, 386.

Altshiller, N., Altshiller-Court, N. See Court, N. A.

Ames, L. D., XXIV, 275.

Archibald, R. C., XXIX, 435.

Baker, R. P., XXII, 283; XXV, 244, 244; XXVII, 302; XXX, 400.

Ballantine, J. P., XXX, 213, 214.

Barnett, I. A., XXV, 243, 244; XXVI, 435, 435; XXIX, 203, 203; XXX, 223.

Barrow, D. F., XXI, 272; XXIII, 300.

Barter, J. D., XXIX, 10.

Barus, C., XXI, 58.

Bateman, H., XXII, 374, 374; XXX, 15.

Bauer, G. N., XXIII, 252.

Beal, F. W., XXI, 483; XXIII, 437; XXV, 436.

Beatty, S., XXII, 482; XXIII, 436; XXVIII, 161.

Beetle, R. D., XXI, 59; XXII, 266.

Bell, E. T., XXIV, 417, 417; XXV, 193, 193, 193, 193, 193, 393, 393; XXVI, $152,152,152,396,396,396$; XXVII, 1, 2, 2, 2, 149, 149, 149, 149, $149,413,414,414,414,414$; XXVIII, 96, 97, 97, 97, 282, 283, 283; XXIX, 11，11，107, 193, 194, 194, 194, 194; XXX, 12, 12, 195, 207, $385,385,386,386,386,386,386$.

Bender, H. A., XXX, 394 .

Bennett, A. A., XXII, 3, 483, 483; XXIII, 208, 252; XXV, 241; XXVI, 146, $146,146,146,244,244,338$; XXVII, 51, 98, 350.

Bernstein, B. A., XXII, 2, 172; XXIV, 175; XXVIII, 96, 282; XXIX, 11, 11, $12,194,436,436 ; \mathrm{XXX}, 205$.

Bianchi, L., XXIII, 436.

Birkhoff, G. D., XXI, 60, 272, 272, 483; XXII, 163, 265, 265; XXIII, 60, 262, 262 ; XXIV, 170; XXV, 51, 435, 435, 435; XXVI, 146, 261, 436; XXVII, 67, 265, 307; XXVIII, 5, 5, 236, 236; XXIX, 101.

Blichfeldt, H. F., XXII, 172; XXIII, 203, 401; XXIV, 417; XXV, 242, 386; XXVI, 396; XXVII, 1, 150, 411; XXVIII, 284; XXIX, 194, 217.

Bliss, G. A., XXI, 287, 287, 430; XXII, 282; XXIII, 252, 388; XXIV, 277; XXV, 386; XXVI, 53, 53, 261; XXVIII, 294; XXIX, 101, 206; XXX, 399.

Blumberg, H., XXI, 217, 287, 287; XXII, 426, 427; XXIII, 209, 209, 252; XXIV, 217, 217; XXV, 243, 387; XXVII, 305, 405; XXIX, 203; XXX, 97.

Bôcher, M., XXI, 59, 59.

Borden, R. F., XXIV, 422; XXVI, 261.

Borger, R. L., XXII, 426; XXVI, 262; XXVII, 98. 
Bouton, C. L., XXIII, 60; XXVI, 245.

Boutroux, P., XXVI, 262, 338.

Brahana, H. R., XXIX, 198, 198.

Brand, L., XXIV, 422; XXX, 230.

Bray, H. E., XXIX, 107, 210; XXX, 488.

Brenke, W. C., XXI, 218; XXVII, 197.

Bridgman, P. W., XXIX, 101.

Brink, R. W., XXII, 483; XXVI, 52.

Brinkmann, H. W., XXVII, 150; XXIX, 106, 215, 215.

Brown, B. H., XXIX, 103, 103.

Brown, E. P., XXIX, 102.

Brown, E. W., XXII, 374; XXIII, 262; XXVI, 50; XXX, 295.

Buchanan, D., XXI, 287, 287; XXII, 282; XXIII, 261, 261, 261; XXV, 51, 243 ; XXVI, 245; XXX, 195, 195.

Buck, T., XXVIII, 95.

Burgess, H. T., XXII, 282, 426; XXIII, 252; XXIV, 277.

Burgess, R. W., XXIII, 59; XXIV, 59; XXVII, 263; XXVIII, 154; XXIX, 108. Bussey, W. H., XXV, 386.

Cairns, W. D., XXV, 51; XXVI, 53.

Cajori, F., XXIV, 59, 59; XXV, 193, 193, 393; XXVI, 152, 396; XXVII, 2, 149, 294, 298, 411, 411; XXVIII, 95, 95, 281, 282, 284; XXIX, 12, 12, $12,101,107,195,195 ; \mathrm{XXX}, 13,13,197,225,387,387,387,387$.

Camp, B. H., XXI, 59, 374; XXII, 483; XXVI, 338, XXVIII, 14; XXIX, 109.

Camp, C. C., XXVIII, 383; XXIX, 124.

Campbell, A. D., XXX, 221.

Campbell, G. A., XXVIII, 94.

Campbell, J. W., XXVII, 52.

Campbell, W. W., XXII, 281.

Candy, A. L., XXIX, 125.

Carlson, S. E., XXVIII, 290, 381; XXX, 396.

Carmichael, R. D., XXI, 430, 430, 431; XXII, 2, 282, 426; XXIII, 388, 388, 388,437 ; XXIV $169,217,276,277,421,422$; XXVI, 53, 53, 53, 146, $146,146,388,388,388$; XXVIII, 152, 287.

Carpenter, A. F., XXI, 287, 430; XXIII, 203; XXVII, 1; XXVIII, 283; XXIX, 195; XXX, 13, 13, 196, 387.

Carr, F. E., XXVIII, 294.

Carslaw, H. S., XXII, 162.

Carver, W. B., XXIV, 266; XXVI, 245; XXVII, 261.

Chao, Y. R., XXV, 393.

Chittenden, E. W., XXI, 430, 430; XXII, 426, 426; XXIII, 388; XXIV, 276; XXV, 146, 386; XXVII, 52, 52, 52, 295, 295, 295; XXVIII, 147, 297; XXIX, 123, 123, 123; XXX, 100, 221.

Clarke, E. H., XXVIII, 383.

Clements, G. R., XXI, 287, 287.

Coble, A. B., XXI, 271, 271; XXII, 2; XXIV, 59; XXV, 386; XXVI, 262 , 262, 388; XXVII, 400; XXVIII, 286; XXIX, 120, 120; XXX, 217, 228.

Cohen, T., XXIII, 262; XXVI, 245.

Cole, F. N., XXI, 272; XXII, 2; XXIII, 158; XXVIII, 380.

Coolidge, J. L., XXI, 272; XXII, 266, 482; XXIII, 59, 262; XXVI, 244; XXVII, 261; XXVIII, 239; XXIX, 106, 106; XXX, 205.

Copeland, L. P., XXVIII, 291.

Court, N. A., XXXII, 2; XXIV, 217, 217; XXVI, 262, 262, 435; XXIX, 442.

Cowley, E. B., XXIX, 9, 115.

Cowling, A. H., XXVIII, 384.

Craig, C. F., XXIX, 107.

Crum, W. L., XXVIII, 13, 14, 156, 292, 292; XXIX, 7, 8, 206.

Cummings, L. D., XXI, 59, 483; XXIV, 169; XXV, 50; XXVIII, 163.

Curtis, M. F., XXIV, 60, 466; XXV, 51; XXVI, 245.

Curtiss, D. R., XXVI, 53, 388; XXVIII, 152, 159, 287, 288. 
Daniell, P. J., XXI, 217; XXIII, 209; XXV, 50, 244.

Dantzig, T., XXII, 427; XXVI, 435.

Daus, P. H., XXVII, 412; XXVIII, 281; XXX, 14.

Davis, E. W., XXI, 218.

Davis, H. T., XXVIII, 298, 299; XXIX, 119, 199; XXX, 227, 399.

Decker, F. F., XXIV, 466.

Dickson, L. E., XXI, 58, 59, 59, 59, 166, 166, 287, 430, 430; XXII, 2; XXIII, 59, 252; XXIV, 175; XXV, 241, 386; XXVI, 435; XXVII, 298, $299,300,300,392,392,407$, 407; XXVIII, 300, 301; XXIX, 120, $121,200,200 ; \mathrm{XXX}, 97,217,228,228$.

Dines, C. R., XXI, 431, 431.

Dines, L. L., XXII, 426; XXIII, 261; XXIV, 59; XXV, 387; XXVI, 53; XXIX, 440, 440 .

Dodd, E. L., XXIV, 60, 276; XXV, 243; XXVI, 262; XXVII, 307; XXVIII, 9, 158, 384; XXIX, 216; XXX, 216.

Douglas, J., XXIII, 436; XXVI, 435; XXVII, 398, 398; XXVIII, 238; XXIX, 4, 5, 209, 209; XXX, 8, 9, 204.

Dresden, A., XXI, 287; XXIII, 59; XXVII, 407; XXVIII, 295; XXIX, 121; $\mathrm{XXX}, 224$.

Dunkel, 0., XXVII, 197; XXVIII, 146.

Durand, W. F., XXVII, 1.

Dushman, S., XXVIII, 152.

Edington, W. E., XXIX, 122, 122, 122; XXX, 218.

Eells, W. C., XXIII, 59; XXX, 388.

Eiesland, J., XXII, 266; XXIII, 59, 60; XXIV, 60; XXVII, 410; XXVIII, 4; XXIX, 443, 443.

Eisenhart, L. P., XXI, 58, 271; XXII, 3, 162, 266, 266, 483; XXIII, 59, 261,436 ; XXIV, 59, 60, 370; XXV, 436; XXVI, 53, 53, 435; XXVII, 350, 393; XXVIII, 3, 154, 238, 379; XXIX, 212, 213; $\mathrm{XXX}, 7,297$.

Elmendorf, A., XXII, 427.

Emch, A., XXI, 287, 483; XXII, 426; XXIII, 261; XXIV, 58, 276, 422, 465; XXV, 244, 436; XXVI, 262; XXVII, 51, 345; XXVIII, 157, 241; XXIX, 149; XXX, 226, 392.

Epperson, C. A., XXI, 483.

Ettlinger, H. J., XXIV, 60, 60, 277; XXVI, 52, 262, 262; XXVII, 197, 295 ; XXVIII, 384.

Evans, G. C., XXI, 59, 272; XXII, 266, 482, 483; XXIII, 83, 209; XXVI, 53, 262; XXVII, 308; XXVIII, 382, 382; XXIX, 210, 444.

Everett, H. S., XXVIII, 158.

Eversull, B. M., XXVIII, 289; XXX, 219.

Feinler, F. J., XXX, 196.

Feldstein, M. M., XXIX, 199.

Field, P., XXVI, 52.

Fields, J.' C., XXIII, 59.

Fine, H. B., XXII, 483.

Fischer, C. A., XXI, 162, 483; XXII, 2, 374; XXIII, 59, 261, 436; XXIV, 267; XXV, 436; XXVI, 245; XXVII, 396; XXVIII, 7, 241, 386.

Fite, W. B., XXI, 60; XXII, 162; XXIII, 436; XXIV, 170, 267; XXV, 434; XXVI, 245; XXX, 204.

Ford, L. R., XXIII, 60; XXVI, 435; XXVII, 52; XXX, 215.

Ford, W. B., XXI, 430; XXII, 426; XXIV, 275; XXV, 243; XXVI, 52; XXVII, 52, 403.

Forsyth, C. H., XXI, 288, 374; XXII, 2, 216; XXIV, 266, 276; XXV, 51; XXVI, 245, 245; XXVII, 260, 260; XXVIII, 156; XXX, 214.

Fort, T., XXII, 265, 426; XXIV, 59; XXVI, 262; XXX, 226.

Foster, M. C., XXIX, 215; XXX, 487. 
Franklin, P., XXV, 435; XXVIII, 384; XXIX, 106, 106, 214, 214; XXX, 482, 482.

Fréchet, M., XXI, 374; XXII, 265; XXIII, 436.

Frizell, A. B., XXI, 217, 271, 287, 374, 431; XXII, 215, 426; XXIII, 60.

Fry, T. C., XXV, 436; XXVIII, 296.

Gaba, M. G., XXIV, 59; XXVII, 198.

Garabedian, C. A., XXIX, 146, 211; XXX, 295.

Garretson, W. V. N., XXIII, 60.

Gehman, H. M., XXX, 401, 483.

Gibbens, G. E. C., XXVII, 305; XXX, 393.

Gillespie, D. C., XXI, 60; XXIV, 60; XXVII, 52.

Gilman, R. E., XXVIII, 12; XXX, 294.

Glashan, J. S. C., XXVIII, $160,160$.

Gleason, R. E., XXII, 266.

Glenn, 0. E., XXI, 58, 374; XXII, 374, 374; XXIII, 158, 158, 261, 436; XXIV, 170, 267, 370, 466; XXV, 50, 435, 435, 435; XXVI, 244, 244, 388; XXVII, 98, 251, 346; XXVIII, 12, 163, 292; XXIX, 110, 151, 208,437 ; XXX, 212, 483.

Gokhale, V. D., XXVIII, 302.

Gossard, H. C., XXII, 216; XXIII, 209.

Gould, A. B., XXV, 242.

Gouwens, C., XXX, 404.

Graustein, W. C., XXI, 272; XXII, 266; XXIV, 60; XXVI, 245, 245; XXVII, 257; XXVIII, 242, 381; XXIX, 104, 104, 212, 441, 441; XXX, 208.

Graves, L. M., XXX, 395.

Green, G. M., XXI, 162, 271, 483; XXII, 3, 162, 162, 266; XXIII, 60, 60, $261,43 i ;$ XXIV, 59,59, 267.

Griffin, F. L., XXX. 15, 388.

Griffiths, L. W., XXX, 196.

Groat, B. F., XXV, 50, 51.

Gronwall, T. H., XXI, 162, 272, 272, 272, 484; XXII, 374, 374, 374, 374, 374; XXIII, $60,61,61,61$; XXV, $244,244,244,244,435$; XXVI, 146, 338, 338; XXVII, 1, 53, 53, 53, 98, 349, 349; XXVIII, 9, 93, 239, 239 ; XXIX, 208, 218, 218.

Grove, C. C., XXIII, 261.

Gummer, C. F., XXVII, 51; XXVIII, 12, 160.

Hale, G. E., XXII, 1.

Hallett, G. H., XXIV, 466; XXV, 146.

Hancock, H., XXV, 243, 244; XXX, 221.

Harding, A. M., XXII, 427; XXIV, 217.

Hardy, G. H., XXII, 215; XXVI, 338.

Hart, W. L., XXI, 287, 430; XXII, 283; XXIII, 261, 261, 437; XXVII, 52, 53, 308, 405; XXVIII, 301.

Haskell, M. W., XXI, 166; XXII, 2, 172; XXIII, 60; XXIV, 417; XXV, 193; XXVI, 152; XXVII, 149, 412.

Haskins, C. N., XXI, 272; XXII, 162; XXV, 51; XXIX, 208.

Hassler, J. 0., XXII, 426.

Hathaway, A. S., XXI, 374; XXII, 426; XXIII, 252.

Hausle, E., XXVIII, 7.

Hazlett, 0. C., XXII, 265; XXIII, 59, 436; XXIV, 267, 369; XXV, 243; XXVI, 146, 245; XXVII, 52, 53; XXVIII, 164; XXIX, 109; XXX, 212.

Hebbert, C. M., XXIII, 388.

Hedrick, E. R., XXI, 218; XXII, 215; XXIII, 209; XXIV, 218; XXVII, 52, 197; XXVIII, 146; XXIX, 127, 437; XXX, 97, 401.

Hewes, L. I., XXIII, 60.

Hildebrandt, T. H., XXII, 283; XXIII, 388, 388; XXIV, 422; XXVI, 53; XXVII, 52, 407.

Hille, C. E., XXVII, 260; XXVIII, 159; XXIX, 6, 7, 7, 208; XXX, 209, 295. 
Hitchcock, F. L., XXIV, 266; XXVII, 397; XXIX, 115.

Hoar, R. S., XXVIII, 296.

Hodge, F. H., XXVIII, 297.

Holleroft, T. R., XXIV, 267; XXVI, 435; XXVIII, 243; XXIX, 147, 217.

Hopkins, J. W., XXIV, 466.

Hopkins, L. A., XXVII, 52.

Hoskins, L. M., XXII, 3; XXIII, 203; XXIV, 175; XXV, 393; XXVI, 396; XXIX, 13.

Howe, H. A., XXVI, 53.

Howland, L. A., XXVI, 244.

Hu, M. T., XXIV, 267.

Huntington, E. V., XXI, 59, 272, 483; XXII, 483; XXIII, 60, 60, 261, 261, 261, 262; XXIV, 60; XXVI, 50; XXVII, 252, 345, 394; XXVIII, 5, 155, 239, 240; XXIX, 207, 207; XXX, 10, 206, 207.

Hurwitz, W. A., XXI, 59; XXIV, 60; XXVII, 256, 397; XXVIII, 156.

Hutchinson, J. I., XXIX, 208.

Ingold, L., XXI, 217; XXII, 215; XXIV, 218; XXVII, 52, 197, 197; XXVIII, 146; XXIX, 127, 437; XXX, 97, 98, 401.

Ingraham, M. H., XXVIII, 153; XXIX, 199.

Irwin, F., XXI, 166; XXII, 172, 172.

Jackson, D., XXI, 271; XXII, 2, 162, 265, 483; XXIII, 59, 59, 436, 436; XXV, 435 ; XXVI, 244, 388; XXVII, 52, 52, 52, 53,53,98, 303, 304, 304, 306, 395, 401; XXIX, 5, 121, 201; XXX, 11, 225, 398, 398, 402.

James, G., XXII, 483; XXIII, 252; XXX, 388.

James, G. O., XXVI, 261.

Jensen, C. M., XXX, 396.

Joffe, S. A., XXII. 374.

Johnson, R. A., XXI, 59.

Johnson, W. W., XXV, 50 .

Johnston, E., XXIII, 203.

Kakeya, S., XXIX, 153.

Karpinski, L. C., XXI, 59.

Kasner, E., XXI, 60, 60, 162, 272, 483, 483; XXII, 265, 482 ; XXIII, 262, 300; XXIV, 170,$466 ;$ XXV 146,$435 ;$ XXVI, 146, 338; XXVII, 53, 98, 253, 254; XXVIII, 4, 92; XXIX, 3, 3, 150; XXX, 209, 212, 487, 487. Kazarinoff, D. C., XXIX, 202, 440; XXX, 208.

Kearney, D. E., XXX, 397.

Kellogg, 0. D., XXII, 216; XXIV, 59, 217; XXVI, 146, 436; XXVII, 294, 307,307 ; XXVIII, 236, 236, 385; XXIX, 207.

Kelly, C. T., XXX, 197.

Kemble, E. C., XXI, 162.

Kempner, A. J., XXII, 282; XXIII, 59, 389, 389, 389; XXIV, 277; XXVI, 52, 53 ; XXVII, 304.

Kendall, C., XXVIII, 145; XXIX, 125.

Kent, F. C., XXX, 197.

Keyser, C. J., XXII, 1, 482; XXIII, 203; XXIV, 266, 266; XXV, 434; XXVI, 244.

King, E. F., XXVI, 245.

Kingston, H. R., XXI, 60, 288; XXIV, 277.

Kircher, E. A. T., XXII, 265.

Kline, J. R., XXI, 484; XXII, 483; XXIII, 60, 158, 261, 300; XXIV, 170, 369,$466 ; \mathrm{XXV}, 435$; XXVI, 389; XXVII, 399; XXVIII, 8, 8, 380; $\mathrm{XXX}, 297,482$.

Koopman, B. O., XXIX, 104.

Kormes, M., XXX. 483.

Kraupner, W., XXX, 220.

Küstermann, W. W., XXII, 2, 283. 
Laber, M., XXIII, 203.

Ladd-Franklin, C., XXV, 51.

Lamson, K. W., XXX, 204.

Lane, E. P., XXIV, 422; XXVI, 52; XXVII, 409; XXVIII, 298; XXIX, 117; $\mathrm{XXX}, 392,392$.

Langer, R. E., XXVIII, 236, 237.

Langman, H., XXVII, 250.

Larew, G. A., XXV, 146; XXVII, 256.

Lasley, J. W., XXVII, 258.

Laves, K., XXV, 242.

Lefschetz, S., XXI, 217, 217; XXII, 215; XXIII, 208, 208, 389; XXIV, 217, 422; XXV, 244; XXVI, 262; XXVII, 198; XXVIII, 146, 147, 379; XXIX, 126, 197, 199; XXX, 98, 229.

Lehmer, D. N., XXI, 166; XXII, 172; XXIII, 401; XXIV, 417, 417; XXV, 193; XXVII, 149, 413; XXIX, 195.

Lennes, N. J., XXVIII, 299, 300, 380.

Le Stourgeon, E., XXVII, 98.

Leuschner, A. 0., XXIII, 262.

Levy, H., XXIX, 213; XXX, 487,

Light, G. H., XXIII, 209.

Linehan, P. H., XXI, 374.

Linfield, B. Z., XXVI, 261; XXIX, 5, 6, 107.

Lipka, J., XXIII, 265; XXVI, 244, 338, 436, 436; XXVII, 98, 98, 250, 394; XXVIII, 157; XXIX, 104, 104, 152, 216, 216; XXX, 9, 9, 210, 211.

Logsdon, M. I., XXVII, 403; XXIX, 119.

Longley, W. R., XXII, 163.

Love, C. E., XẌI, 58, 430; XXVI, 261.

Lovitt, W. V., XXI, 430, 430; XXII, 283.

Lunn, A. C., XXV, 435; XXVI, 262, 386; XXVIII, 3.

McAtee, J. E., XXIV, 217; XXV, 146;

McDonnell, J., XXVII, 348; XXIX, 444.

McEwen, G. F., XXIII, 401; XXVI, 262, 263.

McLennan, J. C., XXVIII, 152.

McMackin, F. J., XXII, 483.

McMahon, J., XXVII, 395.

MacDuffee, C. C., XXVII, 404; XXIX, 111, 111, 209; XXX, 223.

Maclay, J., XXI, 272.

MacMillan, W.D., XXI, 430; XXII, 426, 426 ; XXV, 242; XXVI, 388; XXVII, 302.

MacNeish, H. F., XXVI, 243; XXVIII, 158.

Manneback, C., XXVIII, 242.

Manning, W. A., XXIII, 203, 203, 401; XXIV, 175; XXV, 393; XXVI, 152, 152.

March, H. W., XXX, 224.

Maria, A. J., XXX, 488.

Marriott, R. W., XXIX, 109.

Mason, M., XXVI, 386.

Mason, T. E., XXI, 59, 59; XXIII, 389; XXVI, 388; XXVII, 305.

Mathews, R. M., XXVII, 256; XXIX, 8, 8.

Mathewson, L. C., XXIV, 59; XXV, 50.

Meacham, E. D., XXX, 99, 100.

Merriman, G. M., XXX, 219.

Michal, A. D., XXIX, 210, 444; XXX, 98, 215, 296, 485.

Mickelson, E. L., XXX, 397.

Miles, E. J., XXI, 374.

Miller, A. L., XXIII, 261, 388.

Miller, B. I., XXII, 265.

Miller, G. A., XXI, 59, 272, 287, 287, 431; XXII, 2, 283, 283, 426; XXIII, 59, 262, 389, 437; XXIV, 59, 277, 422, 422; XXV, 50, 243, 387; XXVI, 52, 262, 262, 388; XXVII, 52, 301, 406; XXVIII, 5, 155, 156, 290, 382; XXIX, 110, 111, 199; XXX, 217, 223. 
Milne, W. E., XXII, 266; XXVII, 2; XXX, 388.

Mitchell, H. B., XXIII, 158, 261.

Mitchell, H. H., XXII, 266, 266; XXIII, 262, 262.

Moore, C. L. E., XXII, 265; XXIV, 267, 276, 466, 466; XXVI, 244, 244, 436; XXVII, 261; XXIX, 104.

Moore, C. N., XXI, 430; XXII, 283; XXIII, 59; XXIV, 59, 277, 421, 421; XXV, 243, 244; XXVII, 52; XXVIII, 11, 288, 380; XXIX, 111, 439; $\mathrm{XXX}, 220$.

Moore, E. H., XXI, 430; XXII, 427; XXIII, 59; XXVI, 389; XXVIII, 161, 296.

Moore, R. L., XXI, 483, 483; XXII, 266; XXIII, 158, 300, 436; XXIV, 170, $170,267,369$; XXV, 146, 146, 435; XXVII, 297, 298, 346; XXVIII, 9 , 91, 92, 293, 293, 380; XXIX, 108, 119, 125, 438, 438, 438; XXX, $220,230,299,299,484$.

Mordell, L. J., XXIX, 435.

Morenus, E. M., XXVIII, 242.

Morgan, F. M., XXI, 58, 484.

Moritz, R. E., XXVIII, 96.

Morley, F.. XXIII, 262; XXIV, 267; XXVII, 253.

Morris, F. R., XXVIII, 284.

Morse, H. M., XXIII, 60; XXVI, 244; XXVII, 253; XXVIII, 10; XXIX, 112; $\mathrm{XXX}, 211$.

Moulton, E. J., XXI, 431; XXV, 387.

Moulton, F. R., XXI, 272, 430; XXIII, 252; XXVII, 67; XXIX, 201.

Mullikin, A. M., XXVI, 146; XXVII, 248, 349.

Murnaghan, F. D., XXIX, 442; XXX, 293.

Murray, F. H., XXVIII, 385, 386; XXIX, 116, 116, 116, 149, 441; XXX, 10, 296.

Musselman, J. R., XXVIII, 13.

Myller, A., XXVIII, 381.

Neikirk, L. I., XXVII, 2; XXIX, 195; XXX, 198.

Nelson, A. L., XXII, 426, 426; XXIII, 389; XXIV, 277; XXV, 386; XXVI, 52 ; XXIX, 150.

Noble, C. A., XXVIII, 283.

Nörlund, N. E., XXIX, 212.

Nyswander, J. A., XXX, 394.

Osgood, W. F., XXI, 271; XXII, 162, 162, 266, 483; XXIV, 267; XXVII, 391. Owens, F. W., XXVII, 263, 345, 394.

Page, L., XXVI, 435.

Pell, A., XXI, 484; XXIII, 436.

Pell, A. J., XXIV, 267; XXVI, 146; XXVII, 52; XXX, 7.

Pfeiffer, G. A., XXI, 162; XXII, 162, 483; XXIII, 60; XXIV, 170; XXVI, 244; XXVIII, 240; XXIX, 4, 4, 151; XXX, 213.

Phillips, H. B., XXII, 265; XXIV, 276, 276, 466; XXVI, 244, 244; XXVIII, 152 ; XXIX, 112.

Pierce, T. A., XXII, 172; XXVII, 197.

Pierpont, J., XXVIII, 3.

Pitcher, A. D., XXIII, 262; XXV, 386.

Platt, H. H., XXVI, 261.

Pólya, G., XXX, 10.

Poor, V. C., XXII, 282, 282; XXVII, 393.

Poritsky, H., XXIX, 115.

Porter, M. B., XXII, 216; XXV, 146; XXVIII, 15; XXX, 15.

Post, E. L., XXVI, 435, 435; XXVII, 396; XXIX, 9, 113, 114, 114, 114; $\mathrm{XXX}, 11,207$.

Pounder, I. R., XXVIII, 161.

Press, A., XXVIII, 243. 
Rainich, G. Y., XXIX, 148, 213, 437, 441; XXX, 8, 205, 293, 294, 300, 486. Ranum, A., XXI, 271; XXII, 265.

Rasor, S. E., XXII, 283.

Raynor, G. E., XXIX, 116.

Rayworth, J. C., XXII, 216.

Reaves, S. W., XXII, 216.

Reed, L. J., XXI, 483.

Reilly, J. F., XXVIII, 295.

Reinsch, B. P., XXX, 403.

Reynolds, C. N., XXV, 50; XXVI, 52, 52, 245; XXX, 220.

Rice, L. H., XXIII, 60; XXVI, 52; XXVII, 344; XXVIII, 93; XXIX, 6, 153. Richardson, L. J., XXII, 2.

Richardson, R. G. D., XXI, 60; XXIV, 59; XXVII, 258; XXIX, 439; XXX, 10.

Rider, P. R., XXI, 374; XXII, 374; XXIII, 20॰; XXIV, 217, 369, 422; XXVI, 388; XXVIII, 289; XXX, 397.

Rietz, H. L., XXV, 242, 387; XXVI, 53, 262; XXVII, 198; XXIX, 197; XXX, 391.

Ritt, J. F., XXI, 374, 375, 483; XXII, 265, 265, 482, 482; XXIV, 170, 267, $369,370,465$; XXV, 435; XXVI, .435; XXVII, 98, 347, 348; XXVIII, 90, 91, 241; XXIX, 147; XXX, 216, 298, 298, 486, 486.

Robertson, H. P., XXX, 14.

Robinson, L. B., XXII, 266; XXIII, 436; XXV, 50, 435, 435; XXVIII, 94. Robison, G. M., XXVII, 264.

Roe, E. D., XXIII, 158, 158, 158; XXIV, 59, 59, 59; XXV, 435, 435; XXVI, 52.

Roe, J. R., XXIII, 157; XXIV, 59; XXV, 50.

Roever, W. H., XXII, 215, 216; XXIII, 208; XXIV, 218; XXV, 242; XXVI, 53, 389; XXVIII, 147, 302, XXIX, 126.

Roman, I., XXIX, 201.

Romig, H. G., XXX, 15.

Roos, C. F., XXX, 486.

Rosenbaum, J., XXI, 60.

Rouse, L. J., XXIV, 422.

Rowe, J. E., XXI, 271, 483, 483; XXII, 265; XXIII, 261, 300, 436; XXIV, $58,58,58,170,466 ; \mathrm{XXV}, 50 ; \mathrm{XXVI}, 245,436$; XXVII, 251, 393 ; XXVIII, 13.

Running, T. R., XXII, 426.

Rutledge, G., XXVI, 388; XXIX, 113, 152.

Safford, F. H., XXI, 483; XXIII, 59, 436; XXIV, 466; XXV, 435; XXIX, 212.

Sakellariou, N., XXVI, 436.

Schlesinger, F., XXVI, 50.

Schmidt, K., XXVIII, 381.

Schmiedel, O., XXI, 217; XXVI, 261; XXVII, 197.

Schottenfels, I. M., XXII, 283; XXX, 230, 230.

Schwatt, I. J., XXVII, 261, 262, 262, 262, 262, 348, 348, 348, 348, 400, 400, $400,402,402$; XXVIII, $6,6,6,163,163,244,244,386,387,387$; XXIX, 115, 151 .

Schweitzer, A. R., XXI, 287, 374; XXII, 2, 3, 162, 163, 163, 265, 283, 283, 374, 374, 483; XXIII, $60,60,60,60,252,300,300,388,388$; XXIV, 276, $276,369,369,369,422,422$; XXV, 51, 244, 244, 387, 435; XXVI, 338, 338,435 ; XXVII, 249, 249, $296,296$.

Seely, C. E., XXI, 271; XXIII, 60; XXIV, 466.

Sensenig, W., XXV, 243.

shapley, H., XXIX, 101.

Sharpe, F. R., XXI, 272; XXIII, 59; XXIV, 59, 466; XXVI, 52; XXVII, 52; XXVIII, $379 ;$ XXX, 393.

Shaw, J. B., XXI, 287; XXII, 282; XXIII, 389; XXIV, 277; XXV, 244; XXVI, 53 ; XXVII, 408, 408; XXVIII, 295.

Sheffer, H. M., XXI, 218; XXII, 265, 265, 282, 282.

Sheffer, J. M., XXX, 485 .

Shohat, J. A., XXX, 227, 395, 395. 
Shugert, S. P., XXVI, 52.

Silverman, L. L., XXI, 59, 271; XXII, 265; XXV, 436: XXIX, 112.

Simon, W. G., XXV, 244.

Simonds, E. F., XXIII, 436; XXVI, 146.

Sinclair, M. E., XXIX, 438; XXX, 222, 222.

Sisam, C. H., XXI, 429; XXII, 215, 282, 425; XXIII, 252; XXIV, 421; XXV, $243 ; \mathrm{XXX}, 227$.

Slaught, H. E., XXVIII, 152.

Slobin, H. L., XXIII, 252.

Slocum, S. E., XXIV, 370 .

Smail, L. L., XXV, 393; XXVII, 2, 2; XXIX, 12, 196, 196, 196; XXX, 197.

Small, D., XXX, 197.

Smith, D. E., XXIII, 59.

Smith, D. M., XXII, 425 .

Smith, E. R., XXI, 59; XXVII, 304; XXIX, 126.

Smith, G. W., XXIII, 389; XXIX, 125, 126.

Smith, H. L., XXX, 229.

Smith, M. G., XXIV, 421.

Smith, P. F., XXIII, 262.

Smith, W. M., XXII, 266.

Snyder, V., XXI, 272; XXIII, 59; XXIV, 59, 466; XXVI, 52; XXVII, 52; XXVIII, 379; XXX, 203, 209, 210, 393.

Sommerville, D. M. Y., XXV, 146.

Speiser, A., XXVIII, 294.

Sperry, P., XXII, 427; XXV, 393; XXIX, 196.

Stecker, H. F., XXI, 483.

Steed, D. V., XXVII, 413; XXX, 14.

Steimley, L. L., XXI, 287; XXVI, 262; XXX, 403, 404.

Stone, M. H., XXX, 297, 297.

Story, W. E., XXIII, 262.

Stouffer, E. B., XXI, 430; XXIII, 208, 389; XXV, 243; XXVI, 388; XXVII, 198,$198 ;$ XXVIII, 147,$289 ; \mathrm{XXX}, 99$.

Strachan, R. C., XXI, 483.

Sullivan, C. T., XXI, 430.

Synge, J. L., XXVIII, 7.

Taber, H., XXIII, 262, 300.

Taylor, J. H., XXX, 400.

Taylor, J. S., XXIII, 203; XXV, 393; XXVI, 245; XXVII, 259; XXIX, 105; $\mathrm{XXX}, 210,214,486$.

Thomas, J. M., XXIX, 443.

Thomas, T. Y., XXIX, 218; XXX, 402.

Tolman, R. C., XXVIII, 152.

Turner, J. S., XXVIII, 297 ; XXIX, 127, 202; XXX, 99, 402.

Underhill, A. L., XXI, 287.

de la Vallée Poussin, C. J., XXI, 483; XXII, 2.

Van de Walle, W. E., XXX, 298.

Vandiver, H. S., XXI, 59, 272, 483; XXII, 2, 162, 374; XXIII, 158, 158, 262 ; XXIV, 466, 466, 466, 466; XXV, 436; XXVI, 436; XXVII, 98, 252 ; XXIX, 436, 436; XXX, 228, 229.

Van Vleck, E. B., XXI, 271; XXII, 427; XXIII, 60, 252; XXV, 387; XXVII, 406; XXX, 225.

Veblen, 0., XXIII, 83, 437; XXIV, 170, 170; XXV, 435; XXVI, 436; XXVIII, 154; XXIX, 3, 101, 218; XXX, 402.

Wahlin, G. E., XXI, 430; XXIII, 251; XXIV, 276; XXVI, 388; XXX, 224. Walsh, J. L., XXIII, 389; XXIV, 369; XXVI, 244, 244, 245; XXVII, 51, 52, 
52, 248, 265, 395; XXVIII, 12, 93, 164, 241; XXIX, 102, 102, 150, 211,$442 ; \mathrm{XXX}, 206,207$.

Weaver, J.H., XXI, 484; XXII, 482; XXIII, 158, 436; XXVII, 51.

Webber, W. P., XXVII, 294.

Webster, A. G., XXIII, 59; XXV, 49; XXVIII, 11, 11.

Wedderburn, J. H. M., XXI, 59; XXVI, 338; XXVII, 257, 258; XXX, 487, 488.

Weeks, E. A., XXI, 218.

Weisner, L., XXIX, 8, 113, 151; XXX, 209.

Wells, V. H., XXIII, 389.

West, C. J., XXII, 282.

Westfall, W. D. A., XXVII, 52, 197; XXVIII, 146.

Whelan, A. M., XXIX, 147.

White, H. S., XXI, 59, 162, 483; XXII, 2, 163, 281; XXIII, 300; XXV, 436; XXVII, 392 ; XXIX, 436 ; XXX, 484.

Whittemore, J. K., XXIII, 261, 436; XXIV, 170; XXV, 434; XXVI, 146, 435; XXVII, 393; XXVIII, 90; XXX, 7.

Widder, D. V., XXX, 485.

Wiener, N., XXIII, 261; XXIV, 267; XXVI, 244, 244, 244, 244; XXVII, 255, 255, 255, 397; XXVIII, 15, 164, 291; XXIX, 105, 106, 211, 211, 440; $\mathrm{XXX}, 215,224,482$.

Wilczynski, E. J., XXI, 287, 430; XXII, 281, 283; XXIV, 422; XXV, 243, 386, 386; XXVI, 262, 388; XXVII, 297, 297, 404; XXVIII, 162.

Wilder, C. E., XXII, 483; XXVII, 248; XXVIII, 153, 153.

Wilder, R. L., XXVIII, 299; XXIX, 118, 118, 152; XXX, 226, 484.

Wiley, F. B., XXI, 59 .

Williams, A. R., XXII, 2; XXVIII, 96; XXIX, 13.

Williams, K. P., XXI, 162; XXII, 215, 426; XXVI, 262, 388; XXVIII, 298; XXIX, 124; XXX, 398, 399.

Williams, W. L. G., XXVII, 98; XXVIII, 385.

Wilson, E. B., XXI, 374, 483; XXII, 265, 374, 374, 374; XXIII, 436.

Wilson, W. A., XXII, 483.

Wilson, W. H., XXIII, 388; XXIV, 266.

Winger, R. M., XXIV, 175, 417, 417; XXVI, 152; XXX, 16, 198.

Wong, B. C., XXVIII, 95.

Wood, F. E., XXVII, 409; XXIX, 198, 202.

Woods, R., XXVIII, 294.

Wright, H. N., XXII, 172; XXIII, 401.

Yeaton, C. H., XXII, 427.

Yerkes, R. M., XXVIII, 152.

Young, A. E., XXII, 282.

Young, J. W., XXI, 484.

Zeldin, S. D., XXVI, 245; XXVII, 252, 252, 347; XXVIII, 15, 155, 291, 381; XXIX, 217; XXX, 210. 